\title{
Evaluating seroprevalence to circumsporozoite protein to estimate exposure to three species of Plasmodium in the Brazilian Amazon
}

Virginia Araujo Pereira', Juan Camilo Sánchez-Arcila', Mariana Pinheiro Alves Vasconcelos², Amanda Ribeiro Ferreira', Lorene de Souza Videira', Antonio Teva ${ }^{3}$, Daiana Perce-da-Silva ${ }^{4}$, Maria Teresa Queiroz Marques ${ }^{5}$, Luzia Helena de Carvalho ${ }^{6}$, Dalma Maria Banic ${ }^{4}$, Luiz Cristóvão Sobrino Pôrto ${ }^{5}$ and Joseli Oliveira-Ferreira ${ }^{1 *}$

\begin{abstract}
Background: Brazil has seen a great decline in malaria and the country is moving towards elimination. However, for eventual elimination, the control program needs efficient tools in order to monitor malaria exposure and transmission. In this study, we aimed to evaluate whether seroprevalence to the circumsporozoite protein (CSP) is a good tool for monitoring the exposure to and/or evaluating the burden and distribution of Plasmodium species in the Brazilian Amazon.

Methods: Cross-sectional surveys were conducted in a rural area of Porto Velho, Rondônia state. Parasite infection was detected by microscopy and polymerase chain reaction. Antibodies to the sporozoite CSP repeats of Plasmodium vivax, P. falciparum, and P. malariae (PVCS, PfCS, and PmCS) were detected using the enzyme-linked immunosorbent assay technique. Human leukocyte antigen (HLA)-DRB1 and DQB1 genes were typed using Luminex ${ }^{\circledast}$ XMAP ${ }^{\circledast}$ technology.

Results: The prevalence of immunoglobulin G against P. vivax CSP peptide (62\%) was higher than P. falciparum (49\%) and $P$. malariae (46\%) CSP peptide. Most of the studied individuals had antibodies to at least one of the three peptides (72\%), 34\% had antibodies to all three peptides and $28 \%$ were non-responders. Although the majority of the population was not infected at the time of the survey, $74.3 \%$ of parasite-negative individuals had antibodies to at least one of the CSPs. Importantly, among individuals carrying the haplotypes DRB1*04 DQB1*03, there was a significantly higher frequency of PfCS responders, and DRB1*16 DQB1*03 haplotype for PvCS and PfCS responders. In contrast, $\mathrm{HLA}-\mathrm{DRB} 1^{*} 01$ and $\mathrm{HLA}-\mathrm{DQB} 1^{*} 05$ allelic groups were associated with a lack of antibodies to $P$. vivax and $P$. falciparum CSP repeats, and the haplotype DRB1*01 DQB1*05 was also associated with non-responders, including non-responders to P. malariae.

(Continued on next page)
\end{abstract}

\footnotetext{
*Correspondence: lila@ioc.fiocruz.br

'Laboratory of Immunoparasitology, Oswaldo Cruz Institute/Fiocruz, Av. Brasil

4365, Manguinhos, Rio de Janeiro, Brazil

Full list of author information is available at the end of the article
}

(c) The Author(s). 2018 Open Access This article is distributed under the terms of the Creative Commons Attribution 4.0 International License (http://creativecommons.org/licenses/by/4.0/), which permits unrestricted use, distribution, and reproduction in any medium, provided you give appropriate credit to the original author(s) and the source, provide a link to the Creative Commons license, and indicate if changes were made. The Creative Commons Public Domain Dedication waiver (http://creativecommons.org/publicdomain/zero/1.0/) applies to the data made available in this article, unless otherwise stated. 
(Continued from previous page)

Conclusions: Our results show that in low transmission settings, naturally acquired antibody responses against the CSP repeats of $P$. vivax, $P$. falciparum, and $P$. malariae in a single cross-sectional study may not represent a valuable marker for monitoring recent malaria exposure, especially in an area with a high prevalence of $P$. vivax. Furthermore, HLA class II molecules play an important role in antibody response and require further study with a larger sample size. It will be of interest to consider HLA analysis when using serosurveillance to monitor malaria exposure among genetically diverse populations.

Keywords: Malaria, Circumsporozoite protein, Serological marker, Human leucocyte antigen, IgG antibody, Porto Velho, Rondônia, Brazil

\section{Multilingual abstracts}

Please see Additional file 1 for translations of the abstract into the five official working languages of the United Nations.

\section{Background}

Malaria remains an acute public health problem, particularly in Sub-Saharan Africa, despite an impressive reduction in the number of cases globally. According to the latest estimates from the World Health Organization, there were 212 million new cases of malaria and 429000 deaths in 2015 worldwide [1].

In Brazil, major successful control efforts have contributed to the number of cases declining from 615246 in 2000 to 143145 in 2014, a reduction of 76.7\% [2]. Based on this achievement, the Ministry of Health launched the Plan for the Elimination of Malaria in Brazil, in November 2015 [3]. In 2016, 128503 cases were reported in Brazil, with Plasmodium vivax representing $88.3 \%$, P. falciparum $11.7 \%$, and only a few cases of $P$. malariae being reported (however, molecular techniques have detected this species in $9-12 \%$ of malaria patients in selected settings) $[4,5]$. While the prospects for eliminating P. falciparum malaria are good, a progressive increase in $P$. vivax cases remains a challenge $[6,7]$. In $P$. vivax infections, the presence of asymptomatic carriers and the possibility of relapse impose additional challenges for surveillance. In addition, there is no current method for diagnosing hypnozoite carriers and P. vivax infections that recur after drug treatment can be attributed to relapse arising from the dormant liver stages (hypnozoites) or recrudescence originating from asexual blood-stage parasites that survived drug treatment, or a reinfection resulting from a new mosquito inoculation [8].

The challenge to sustain and ensure malaria elimination is to detect asymptomatic infections in order to block transmission. In endemic areas, the risk of infection has been routinely estimated by parasite prevalence using microscopy and rapid diagnostic tests (RDTs) or estimated using the entomological inoculation rate (EIR) $[9,10]$. Microscopy and RDT are easy to deploy, but are not sensitive enough to detect low-grade infections that are typical of settings with residual malaria transmission. The EIR method is time consuming, requires very large mosquito samples, and has been found to be less accurate in low transmission areas [11]. Many studies have been reporting the evaluation and validation of polymerase chain reaction (PCR) assays for the detection of malaria in the field. However, costs and technical requirements currently hamper their implementation in endemic areas [12].

Prior to the molecular diagnosis era, serology was used to identify subjects with evidence of current or recent infection, with this method having the potential to detect not only ongoing blood stage infections but also symptomatic or asymptomatic infections [13-15]. In recent years, using serological data to monitor $P$. falciparum and $P$. vivax transmission intensity and exposure has gained recognition in the monitoring of changes in transmission, and in identifying hotspots of transmission and high-risk groups [16-19].

Numerous studies conducted in a variety of epidemiological settings have revealed that the levels and seroprevalence of sporozoite antibodies, especially those against the repetitive epitope of circumsporozoite protein (CSP) of P. falciparum, are a predictor of parasite exposure over time and are a good indicator of malaria transmission intensity [20-24]. However, the persistence of antibody responses long after malaria transmission has ceased has been reported in a non-endemic region of Brazil and in a low transmission area in Thailand, and long-lived anti-CSP antibody responses have been detected in the absence of a blood-stage infection $[25,26]$. Moreover, antibody responses to sporozoite showed some evidence that host genetics, such as human leukocyte antigen (HLA) class II, do affect antibody responses [27-29].

In naturally exposed individuals in the Brazilian Amazon, the expression of HLA-DR7 has been associated with poor responses against the CSP repetitive region of $P$. vivax (VK210), HLA-DR3, and HLA-DR5, with poor responses against $\mathrm{N}$-terminal region [28-30]. In a phase I trial of $P$. falciparum multiple antigen peptide vaccine, the 
formulation elicited high levels of parasite-specific antibodies in volunteers expressing DQB1*0603, DRB1*0401, or DRB1*1101 class II molecules [31]. HLA class II molecules may play an important role in antibody response and show huge variability. In humans, this will need to be considered when employing serosurveillance.

In Brazil, a number of cross-sectional studies have aimed to characterize antibody response to CSPs of all three Plasmodium species: P. falciparum, P. vivax, and $P$. malariae. Most of the studies on the antibody responses to CSPs of $P$. vivax and P. falciparum point out an association with malaria exposure [24, 32].

In this study, we aimed to test whether seroprevalence to CSPs of $P$. falciparum, $P$. vivax, and $P$. malariae is a good tool for monitoring the exposure to and/or evaluating the burden and distribution of these species in a rural area of Porto Velho, Rondônia state. This endemic area has experienced a decline in $P$. falciparum cases, a progressive increase in $P$. vivax cases, and has had a few reported cases of $P$. malariae. The influence of HLADRB1 and HLA-DQB1 allelic products on the antibody responses was also taken into consideration.

\section{Methods}

\section{Study population and sample collection}

This study comprised of cross-sectional surveys being conducted in a rural community of Porto Velho (63'54' $\left.13^{\prime \prime} \mathrm{W} 8^{\circ} 45^{\prime} 43^{\prime \prime} \mathrm{S}\right)$, municipality of Rondônia state, Brazilian Amazon.

In this region, malaria transmission occurs throughout the year with seasonal fluctuations and an increased number of cases in the dry months between April and September. Anopheles darlingi is the main vector in all Amazon areas and is the dominant species in Rondônia [33-35]. The numbers of malaria cases in Rondônia were 43514 in 2010 and 30412 in 2011 (when the survey was conducted). In both years, $P$. vivax was the predominant species, accounting for more than $88 \%$ malaria cases, there were $10.8 \%$ of $P$. falciparum cases in 2010 and 7.2\% in 2011, and no $P$. malariae cases were reported [36].

Samples and survey data were collected during the dry months of June-September in 2010 and 2011. Study participants were recruited via visits to their houses, which were randomly selected. Those who were diagnosed at the outpatient clinic were also recruited. The enrolment exclusion criteria were as follows: age $<10$ years old, pregnancy, breastfeeding, antimalarial drug use, mental disorders, and status as a member of an indigenous population. Informed consent was obtained from all participants and the protocol of this study was reviewed and approved by the Fundação Oswaldo Cruz Ethical Committee (CEP/ FIOCRUZ, 492/08).
All individuals answered a questionnaire and were asked if they had any symptoms, such as fever, headaches, chills, myalgia, and/or nausea. After the epidemiological survey was conducted, blood was drawn from each participant by venipuncture and malaria infection was diagnosed by microscopic examination of Giemsa-stained blood smears and PCR using primers specific for genus (Plasmodium spp.) and species ( $P$. falciparum, $P$. vivax, and $P$. malariae). The amplification protocols have been described previously [37].

Subjects were considered positive for Plasmodium infection if they were positive according to the thick blood smear and/or PCR. The parasitological evaluations were performed by examination of 200 fields at 1000fold magnification under oil immersion. Parasite densities were estimated by counting the number of parasites per microliter of blood (all species and stages) per 200 leukocytes in thick blood films, multiplying this by the number of individual leukocytes, and dividing this by 200. All smear-positive individuals were treated with antimalarial drugs, as according to the protocol of the Brazilian Ministry of Health.

\section{Peptide synthesis}

Synthetic peptides representing the repeated immunedominant epitope of the CSP were used as an antigen for the detection of immunoglobulin G (IgG) antibodies. For $P$. vivax, all three variant sequences were used: $P$. vivax VK210 DGQPAGDRAAGQPAG-(DRADGQPAG) $P$. vivax VK247 (ANGAGNQPG) 3 -ANGAGN, and $P$. vivax-like (APGANQEGGAA) ${ }_{3}$. For $P$. falciparum and $P$. malariae, sequences $(\mathrm{NANP})_{8}$ and (GNAA) ${ }_{2}$-GNDA$(\text { GNAA })_{4}$ were used, respectively.

\section{Measuring IgG responses}

The enzyme-linked immunosorbent assay (ELISA) for the detection of antibodies to the CSP was conducted, as previously described [38]. Briefly, MaxiSorp ${ }^{\mathrm{TM}}$ plates (Nunc, Carlsbad, CA, USA) were coated with $5 \mu \mathrm{g} / \mathrm{ml}$ of the synthetic peptides representing CSP repeats of $P$. vivax (PvCS), P. falciparum (PfCS), and P. malariae (PmCS), and incubated overnight at $4{ }^{\circ} \mathrm{C}$. Individual plasma samples were assayed at a dilution of 1:100 for one hour at $37^{\circ} \mathrm{C}$. Bound antibodies were detected by IgG-peroxidase conjugated goat anti-human IgG (Sigma-Aldrich, St Louis, MO, USA), followed by O-phenylenediamine dihydrochloride (Sigma-Aldrich, St Louis, MO) and hydrogen peroxide. The optical densities (ODs) were recorded on an ELISA reader (BioTek ELx800 ${ }^{\mathrm{Tm}}$ ) at $490 \mathrm{~nm}$. On each plate, reactive standard sera and non-reactive standard sera from individuals living in non-endemic areas who have never been exposed to malaria infection were placed. Results were expressed as reactivity index (RI), which was 
calculated by dividing the mean OD values of tested samples by the mean plus three standard deviations (SDs) of negative controls tested simultaneously. Samples with an RI greater than 1 were considered positive for the CSP peptide tested. In the case of $P$. vivax, positive samples presenting RI $>1$ for any of the three CSP repeats representing $P$. vivax CSP genotypes (VK210, VK247, and $P$. vivax-like) were considered.

\section{HLA genotyping}

Genomic DNA was isolated from whole blood with ethylenediaminetetraacetic acid (EDTA) using QIAamp ${ }^{\circ}$ DNA Blood Midi Kit (QIAGEN, Chatsworth, CA, USA). The concentration and quality of all DNA were analysed using NanoDrop ${ }^{\circ}$ ND-1000 (Thermo Fisher Scientific, Inc., Waltham, MA, USA). HLA-DRB1 and HLA-DQB1 genes were typed using a Luminex ${ }^{\circ}$ Multianalyte profiling system (One Lambda, Inc., Canoga Park, CA, USA). This system uses LABType SSO One Lambda typing kit (One Lambda, Inc., CA, USA) and is based on the PCR sequence-specific oligonucleotide probes. The highly polymorphic exons 2 of the HLADRB1 and HLA-DQB1 genes were amplified using the kit-specific primer pairs. The $5^{\prime}$ ends of the upstream primers were labeled with biotin. Hybridized amplicons were labeled with streptavidin, R-phycoerythrin, and quantified on the Luminex $100^{\mathrm{m}}$ flow analyzer (One Lambda, Inc., CA, USA). The hybridized patterns were classified using HLA Visual 2.0 software (One Lambda, CA, USA) according to the manufacturer's instructions.

\section{Statistical analysis}

Analysis were done using Epi Info ${ }^{\text {tw }} 2002$ (CDC, Atlanta, GA, USA), GraphPad Prism version 7.0 (GraphPad Software, Inc., San Diego, CA, USA), and $\mathrm{R}$ version 3.4.0 (Vienna, Austria) [39]. Differences in medians in terms of the study population data were calculated using the non-parametric Mann-Whitney U test. The student's ttest was used to compare the means of normally distributed data, or normalized transformations were performed on raw data before testing by one-way analysis of variance (ANOVA). Differences in the proportions of the frequencies between variables were evaluated using the chi-square $\left(\chi^{2}\right)$ test. Associations between antibody levels and epidemiological data were assessed with Spearman's rank correlation. Overall associations of antibody responses with the alleles from each HLA-DRB1* and HLA-DQB1* loci were evaluated by comparing the allele frequencies between seronegative (non-responders) and seropositive (responders) subjects using a Fisher's exact test $2 \times 2$ contingency table. Statistical significance was set at $P<0.05$.

\section{Results}

\section{Characteristics of the study participants}

Details about the study population are summarized in Table 1. Three hundred and fifty-seven individuals were enrolled in this study. The majority of individuals included in this study were randomly selected $(n=285)$. Only 72 individuals were recruited from the outpatient clinic, but they lived in the same area as the other participants and had similar exposure to malaria infections.

Table 1 Epidemiological characteristics of study participants

\begin{tabular}{|c|c|c|c|c|c|}
\hline & & \multicolumn{2}{|c|}{ Malaria diagnosis } & \multirow[b]{2}{*}{ Total } & \multirow{3}{*}{$P$-value } \\
\hline & & Positive & Negative & & \\
\hline & & $n=98$ & $n=259$ & $n=357$ & \\
\hline \multirow[t]{2}{*}{ Gender $n(\%)$} & Female & $30(19.4)$ & $125(80.6)$ & $155(43.4)$ & 0.003 \\
\hline & Male & $68(33.7)$ & $134(66.3)$ & $202(56.6)$ & \\
\hline Age (years) & & $30.2 \pm 11.6$ & $30.5 \pm 15.5$ & $30.4 \pm 14.5$ & 0.862 \\
\hline Time of residence in endemic area & & $25.6 \pm 11.5$ & $24.2 \pm 13.1$ & $24.5 \pm 12.7$ & 0.352 \\
\hline Previous malaria infection & & $7.7 \pm 12.4$ & $11.7 \pm 15.2$ & $10.6 \pm 14.5$ & 0.021 \\
\hline Time since last malaria infection & & $29.7 \pm 69.4$ & $41.6 \pm 66.7$ & $38.3 \pm 67.5$ & 0.137 \\
\hline \multicolumn{6}{|l|}{ Previous Plasmodium species } \\
\hline P. vivax & & $35(47.3)$ & $71(32.0)$ & $106(35.8)$ & \\
\hline P. falciparum & & $1(1.4)$ & $13(5.9)$ & $14(4.7)$ & 0.236 \\
\hline P. vivaxIP. falciparum & & $38(51.3)$ & $137(61.6)$ & $175(59.1)$ & \\
\hline \multicolumn{6}{|l|}{ Current Plasmodium species } \\
\hline P. vivax & & - & - & $71(72.5)$ & \\
\hline P. falciparum & & - & - & $26(26.5)$ & $<0.0001$ \\
\hline P. vivaxIP. falciparum & & - & - & $1(1.0)$ & \\
\hline
\end{tabular}

Gender: $n$ (\%); Age: mean $\pm S D$; Time of residence in endemic area (years): mean $\pm S D$; Previous malaria infection: mean $\pm S D$; Time since the last malaria infection (months): mean $\pm S D$; Previous Plasmodium species: $n$ (\%); Current Plasmodium species: $n$ (\%) 
Parasite prevalence determined by microscopy and/or PCR was $27 \%$ (71 had P. vivax, 26 had P. falciparum, and one had a mixed infection). It is worth noting that all individuals testing positive by microscopy were also positive by PCR, except five individuals who were found positive only by PCR.

The proportion of males to females was not significant, but the infection rate was higher in males. No differences were observed in the mean age, time of residence in endemic area, and time elapsed since the last malaria episode between parasite-positive and parasite-negative individuals. However, individuals who were parasite-negative recalled a higher number of previous malaria episodes than those who were parasitepositive. The studied population recalled, by memory, having had previous episodes with confirmed diagnosis of $P$. vivax or $P$. falciparum malaria, but no history of $P$. malariae.

Malaria-associated symptoms were present in $86.7 \%$ of the infected individuals, even though they all reported repeated malaria infections. The most common symptoms reported by the participants at the time of blood collection were fever (66\%), headaches (76\%), chills (54\%), myalgia (57\%), and nausea (43\%).

\section{Naturally acquired antibody responses against the CSP}

The prevalence of naturally acquired antibodies to PvCS, PfCS, and PmCS was measured. Of the study participants, $72 \%$ presented IgG against at least one of the three peptides. The prevalence of IgG against PvCS (62\%) was higher than PfCS $(49 \%, P<0.01)$ and PmCS $(46 \%, P<0.001)$. Most of the individuals had antibodies to at least one of the three peptides (72\%), 34\% had antibodies to all three peptides and $28 \%$ were non-responders. Although $P$. malariae was not detected by microscopy or PCR in the study population, the prevalence of antibodies to PmCS (46\%) was similar to PfCS (49\%) (see Fig. 1a). Levels of antibodies, expressed as the RI, were significantly higher in responders to PfCS than to PvCS (see Fig. 1b). However, the IgG RI of anti-CS antibody was also not associated with time of residence in endemic area $(r=0.048 P=0.36$ for PvCS; $r=0.04, P=0.45$ for PfCS, and $r=0.058, P=0.27$ for PmCS) or number of
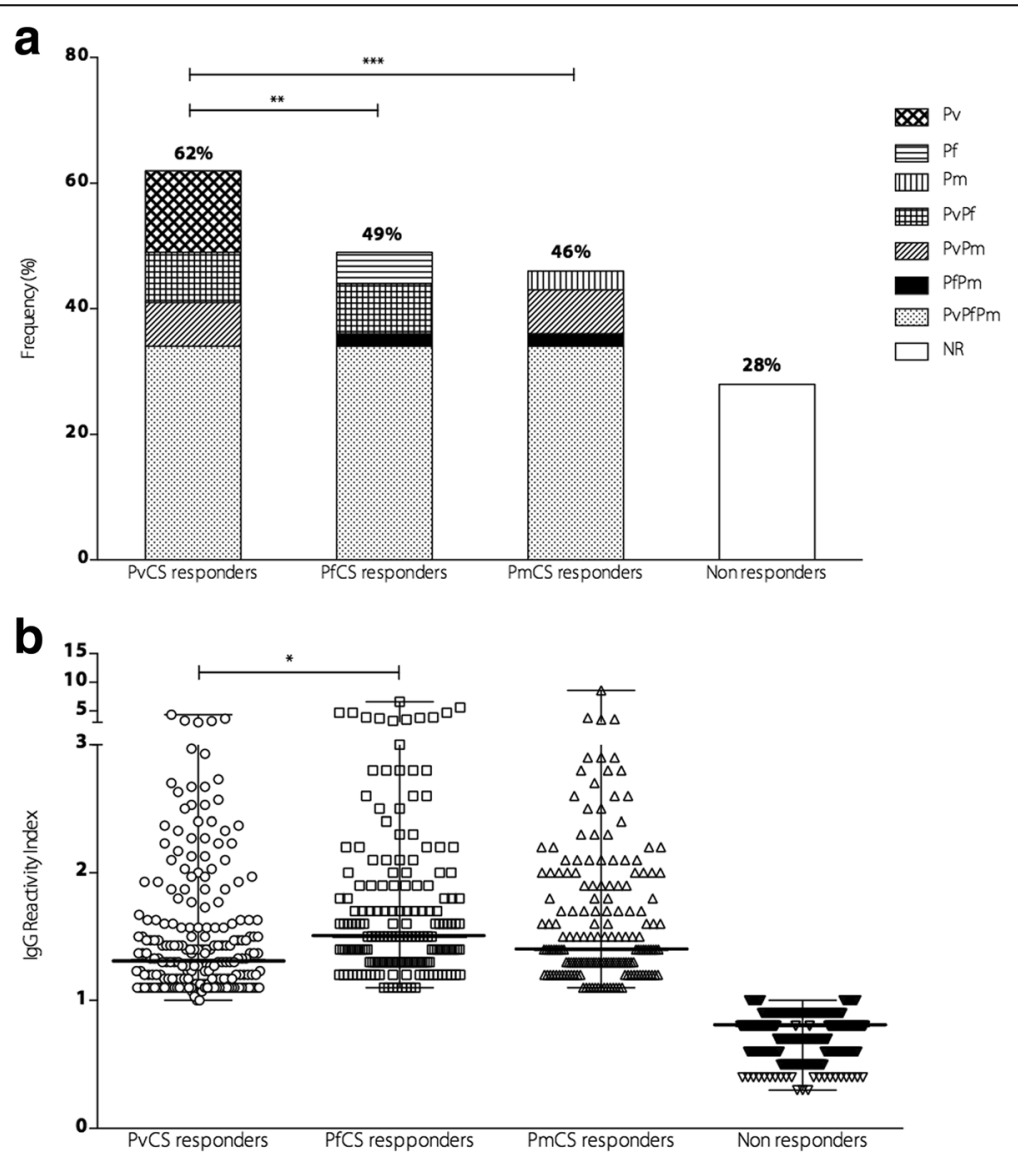

Fig. 1 Prevalence and magnitude of antibodies to CSP repeats. a Frequency of responders to PvCS, PfCS, and PmCS. Pv represents individuals responding to PvCS peptide only, Pf for PfCS only, Pm for PmCS only, PvPf for both PvCS and PfCS peptides, PvPm for both PvCS and PmCS peptides, PfPm for both PfCS and PmCS peptides, and PvPfPm responders to all three peptides. NR represents non-responders to all three peptides. $\mathbf{b}$ Rl of lgG antibodies against each CS peptide. Rl > 1 was considered positive. The bars indicate the median and the range 
past malaria infections $(r=0.016, P=0.75$ for PvCS; $r=0.010, P=0.84$ for PfCS, and $r=0.053, P=0.31$ for PmCS). We did not find any association between antibody responses and age $(r=0.006, P=0.11$ for PvCS; $r=0.009, P=0.06$ for PfCS, and $r=0.004, P=0.21$ for PmCS).

\section{Effect of $P$. falciparum and $P$. vivax infections on $\lg G$ positivity and magnitude}

The prevalence and magnitude of specific CSP antibodies in parasite-positive individuals showed that $51.4 \%$ of $P$. vivax- and $62.9 \%$ of $P$. falciparum-infected individuals had antibodies to the corresponding CSP repeats. In individuals diagnosed with $P$. vivax, the prevalence of antibodies to PvCS was similar to PfCS and PmCS, and $41.7 \%$ were non-responders. In individuals diagnosed with $P$. falciparum, the prevalence of antibodies to PfCS was also similar to PvCS and PmCS. However, only $14.8 \%$ were non-responders, and the prevalence of antibodies to PfCS and PmCS were significantly higher in individuals with $P$. falciparum when compared to $P$. vivax-infected individuals. Although the majority of the population (72\%) was not infected at the time of the survey $(n=257), 74.3 \%$ of the parasite-negative individuals had antibodies to at least one of the CSPs. Among the parasite-negative individuals, the prevalence of antibodies to PvCS was significantly higher when compared to PfCS $(P<0.01)$ and PmCS $(P<0.0001)$, and $25.7 \%$ were non-responders (see Fig. 2a). Comparing the antibody levels between malariapositive and malaria-negative individuals, the presence of parasites did not have any impact on the IgG RI of responders. Likewise, no significant differences were observed in the magnitude of PvCS antibodies in $P$. vivax parasite-positive individuals and PfCS antibodies in $P$. falciparum parasite-positive individuals. Although individuals who were parasite-negative seemed to have higher antibody levels, no significant differences were observed between parasite-positive and parasite-negative individuals for all three CSPs (see Fig. 2b).

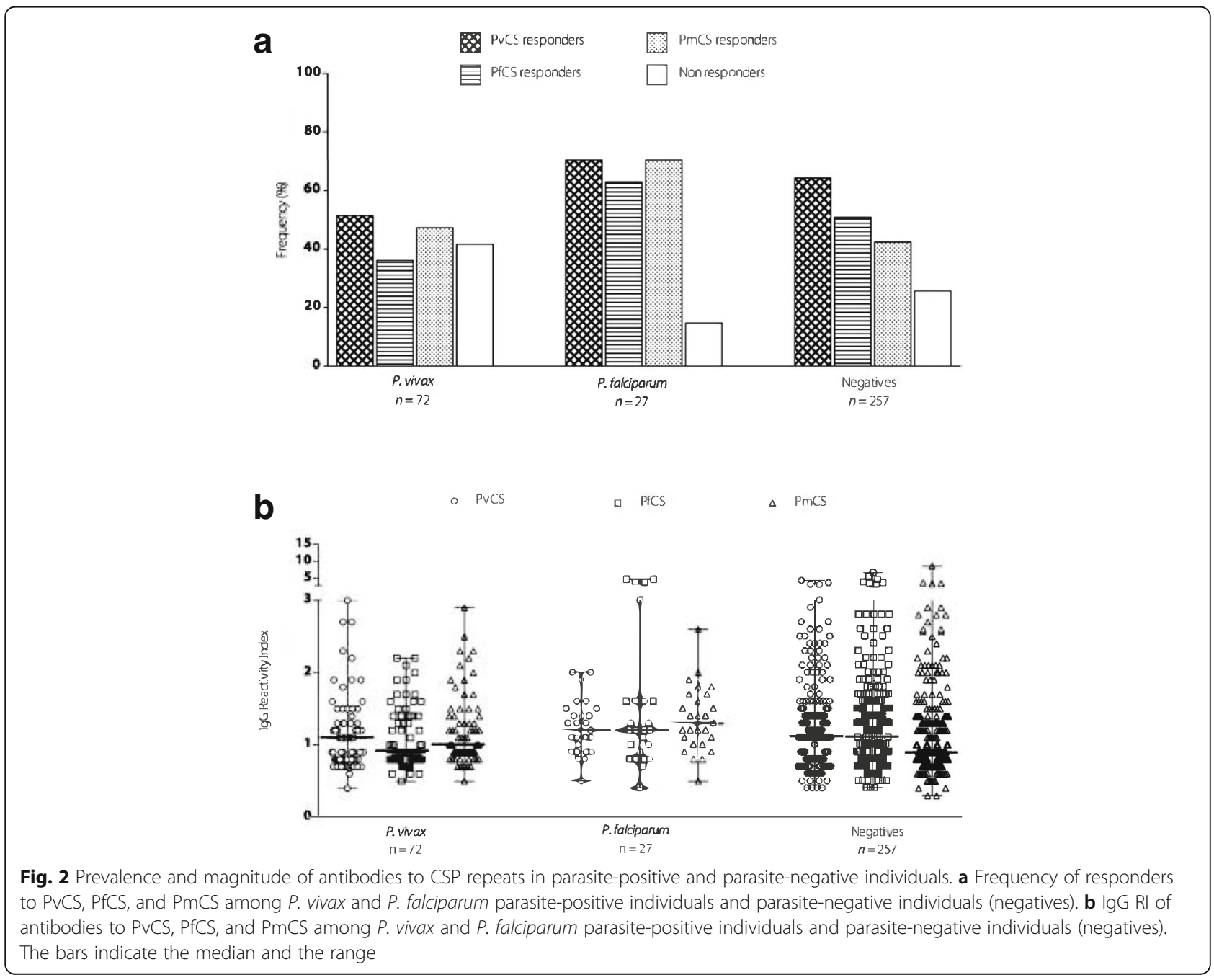


Antibody responses to CSP according to the time elapsed since the last malaria infection

To investigate the ability of the CSP antibody to determine whether individuals had been infected in the recent past, we compared the prevalence and the RI of antiCSP antibodies according to the time elapsed since the individuals' last malaria infection according to their recall in memory.

Individuals positive for malaria were assigned as having malaria in the last $\leq 1$ month. Figure 3 a shows that the frequency of responders to PvCS, PfCS, and PmCS did not significantly differ between individuals who had been infected more recently $(\leq 1$ month and $(1,12]$ months). In individuals who had the last malaria infection over a year ago ( $>12$ months), the prevalence rates of antibodies to PvCS and PfCS were higher when compared with individuals more recently infected. The IgG RI to PvCS, PfCS, and PmCS did not differ according to the time since the last malaria infection. It is noteworthy that among individuals who reported their last malaria infection over the past 12 months, IgG levels to PfCS and PmCS were higher when compared to PvCS (see Fig. 3b). However, no association was observed between the IgG RI and time elapsed since the last malaria infection for all the CSP antigens $(r=0.009, P=0.85$ for PvCS; $r=0.015, P=0.78$ for PfCS, and $r=0.024, P=0.64$ for PmCS).

HLA-DRB1 and HLA-DQB1 allele and antibody responses Considering that HLA class II molecules play an important role in antibody response and that $28 \%$ of individuals did not respond to all three CSP peptides, we evaluated the influence of HLA-DRB1 and HLADQB1 alleles on the naturally acquired IgG responses to the CSPs of $P$. vivax, $P$. falciparum, and $P$. malariae in 280 individuals. The frequency of each allele in responders and non-responders to all three CSPs are summarized in Table 2.

Common allele groups ranged from 13 at the HLADRB1 locus and five at the HLA-DQB1 locus. The predominant HLA-DRB1 allelic groups were HLA-DRB1*04 (16\%) and HLA-DRB1*13 (14\%), and the most frequent HLA-DQB1 were HLA-DQB1*03 (38\%) and HLADQB1*06 (21\%). Although $72 \%$ of the population were positive to at least one of the peptides, the frequency of HLA-DRB1*01 was significantly higher in non-responders to PvCS and PfCS and HLA-DQB1*05 in non-responders to PvCS and PfCS. In contrast, we observed a higher frequency of HLA-DQB1*02 genotype in responders to PfCS peptide. Antibodies to PmCS showed no association with particular HLA-DRB1 and HLA-DQB1 genotypes.

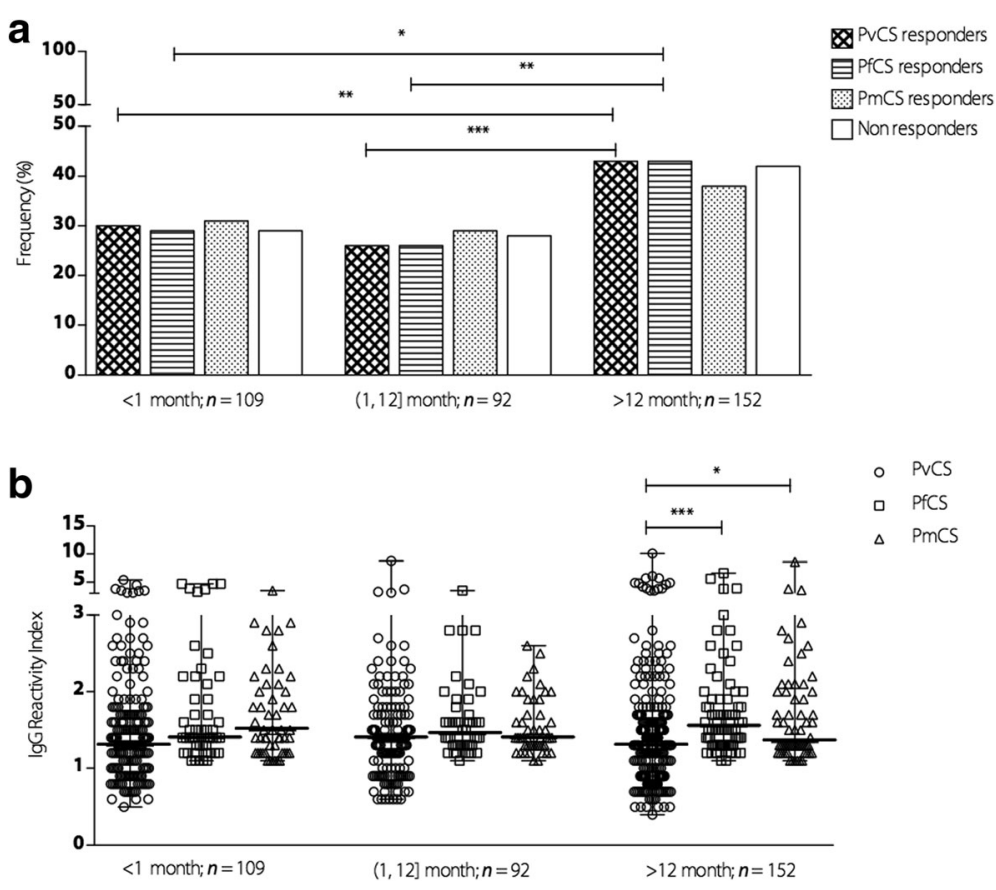

Fig. 3 Prevalence and magnitude of antibodies against CSP repeats according to time since the last malaria infection. a Frequency of responders to PvCS, PfCS, and PmCS of individuals who recalled the last malaria infection within less than 1 month: $<1$ month, more than 1 month and less than 12 months: $(1,12]$ months, and more than 12 months: > 12 months. b lgG RI of antibodies to PvCS, PfCS, and PmCS of individuals who recalled the last malaria infection within less than 1 month: $<1$ month, more than 1 month and less than 12 months: $(1,12]$ months, and more than 12 months: $>12$ months. The bars indicate the median and the range 
Table 2 Frequency $(f)$ and number (n) of antibody responders (R) and non-responders (NR) to CS peptide repeats for $P$. vivax, $P$. falciparum, and P. malariae according to HLA-DRB1 and HLA-DQB1 genotypes

\begin{tabular}{|c|c|c|c|c|c|c|}
\hline & \multicolumn{2}{|l|}{ PvCS } & \multicolumn{2}{|l|}{ PfCS } & \multicolumn{2}{|l|}{ PmCS } \\
\hline & $R$ & NR & $R$ & $N R$ & $R$ & NR \\
\hline & $f(n)$ & $f(n)$ & $f(n)$ & $f(n)$ & $f(n)$ & $f(n)$ \\
\hline \multicolumn{7}{|l|}{ HLA-DRB1 } \\
\hline $\mathrm{DRB} 1 * 01$ & $0.05(18)$ & $0.15(30) * * *$ & $0.04(12)$ & $0.13(36)^{* * *}$ & $0.06(15)$ & $0.11(33)$ \\
\hline $\mathrm{DRB} 1 * 03$ & $0.06(23)$ & $0.05(9)$ & $0.06(17)$ & $0.05(15)$ & $0.06(16)$ & $0.05(16)$ \\
\hline $\mathrm{DRB} 1 * 04$ & $0.16(59)$ & $0.15(29)$ & $0.18(52)$ & $0.13(36)$ & $0.14(35)$ & $0.17(53)$ \\
\hline $\mathrm{DRB} 1 * 07$ & $0.12(43)$ & $0.08(16)$ & $0.13(36)$ & $0.08(23)$ & $0.11(27)$ & $0.10(32)$ \\
\hline $\mathrm{DRB} 1{ }^{*} 08$ & $0.07(27)$ & $0.10(19)$ & $0.09(25)$ & $0.08(21)$ & $0.09(23)$ & $0.07(23)$ \\
\hline DRB 1*09 & $0.02(9)$ & $0.03(6)$ & $0.02(6)$ & $0.03(9)$ & $0.02(6)$ & $0.03(9)$ \\
\hline $\mathrm{DRB} 1 * 10$ & $0.03(12)$ & $0.02(3)$ & $0.01(4)$ & $0.04(11)$ & $0.03(7)$ & $0.03(8)$ \\
\hline $\mathrm{DRB} 1 * 11$ & $0.11(40)$ & $0.10(19)$ & $0.10(27)$ & $0.12(32)$ & $0.12(31)$ & 0.09 (28) \\
\hline $\mathrm{DRB} 1 * 12$ & $0.01(2)$ & $0.03(5)$ & 0.01 (3) & $0.01(4)$ & $0.01(3)$ & $0.01(4)$ \\
\hline DRB1*13 & $0.15(56)$ & $0.11(22)$ & $0.15(41)$ & $0.13(37)$ & 0.14 (34) & $0.14(44)$ \\
\hline DRB1*14 & $0.04(16)$ & $0.04(7)$ & 0.05 (13) & $0.04(10)$ & $0.04(9)$ & $0.05(14)$ \\
\hline $\mathrm{DRB}^{*}{ }^{* 15}$ & 0.09 (33) & $0.10(19)$ & $0.08(23)$ & $0.10(29)$ & $0.10(26)$ & $0.08(26)$ \\
\hline $\mathrm{DRB} 1 * 16$ & $0.08(28)$ & $0.05(10)$ & $0.08(23)$ & 0.05 (15) & 0.07 (18) & $0.06(20)$ \\
\hline \multicolumn{7}{|l|}{ HLA-DQB1 } \\
\hline $\mathrm{DQB} 1{ }^{*} 02$ & $0.17(62)$ & $0.12(24)$ & $0.19(53)^{*}$ & $0.12(33)$ & $0.17(43)$ & $0.14(43)$ \\
\hline $\mathrm{DQB} 1{ }^{* 03}$ & $0.40(145)$ & $0.34(65)$ & $0.41(116)$ & $0.34(94)$ & $0.38(96)$ & 0.37 (114) \\
\hline DQB1 ${ }^{* 04}$ & $0.08(30)$ & $0.08(16)$ & $0.07(21)$ & 0.09 (25) & $0.06(16)$ & $0.10(30)$ \\
\hline $\mathrm{DQB} 1 * 05$ & $0.15(54)$ & $0.24(47) * *$ & $0.13(36)$ & $0.23(65) *$ & $0.17(42)$ & $0.19(59)$ \\
\hline $\mathrm{DQB} 1 * 06$ & $0.20(74)$ & $0.22(42)$ & $0.20(55)$ & $0.22(61)$ & $0.21(52)$ & $0.21(64)$ \\
\hline
\end{tabular}

The association was verified sing a Fisher's exact test $2 \times 2$ contingency table; ${ }^{*} P<0.01,{ }^{* *} P<0.001,{ }^{* * *} P<0.0001$. Each individual contributed with two HLA allele observations

\section{Evaluation of HLA-DRB1 and HLA-DQB1 haplotypes and antibody responses}

Associations attributed to individual DRB1 and DQB1 allele groups were partially reflected by the DRB1 DQB1 haplotypes. These allele groups contributed to 10 common DRB1 DQB1 haplotypes.

Frequencies of responders and non-responders to each peptide according to the haplotypes are described in Fig. 4. The association between DRB1*01 and DQB1*05 of non-responders was mostly due to DRB1*01 DQB1*05 haplotype for PvCS, PfCS, and PmCS. The positive association of DQB1*02 with responders to PfCS was mainly because of the DRB1*07 DQB1*02 haplotype. Among individuals carrying the haplotypes DRB1*04 DQB1*03, there was a significantly higher frequency of responders to PfCS, and DRB1*16 DQB1*03 haplotype for PvCS and PfCS.

\section{Discussion}

Malaria elimination is on the agenda of the control program in Brazil, and effective monitoring of disease transmission is crucial. From 2007 to 2017, P. falciparum has been decreasing (under 11\%), P. vivax has become the predominant species (88\%), and only a few cases of $P$. malariae have been reported in Rondônia state. This epidemiological situation gave us the opportunity to investigate whether seroprevalence to the CSPs of $P$. falciparum, $P$. vivax, and $P$. malariae is a good tool for monitoring the exposure to and/or evaluating the burden and distribution of these species in a rural area of Porto Velho, Rondônia.

Our results show that antibody responses against the CSP repeats of $P$. vivax and $P$. falciparum were higher than those estimated based on blood analysis and/or PCR, and revealed the presence of anti-CSP antibody to P. malariae. This is not surprising as a high prevalence of anti-CSP antibody to $P$. malariae has been reported in five states of the Amazon, including Rondônia [32]. Although we did not detect $P$. malariae infection by PCR, this species seems to be more common than suggested by official data based on microscopy. Studies using nested PCR have found significantly more cases of P. malariae infection in patients from Rondônia (10\%) and Mato Grosso (11.9\%) [4, 5, 32]. In addition, case reports documented that $P$. malariae has the ability to persist in a single host for decades as a chronic, low- 

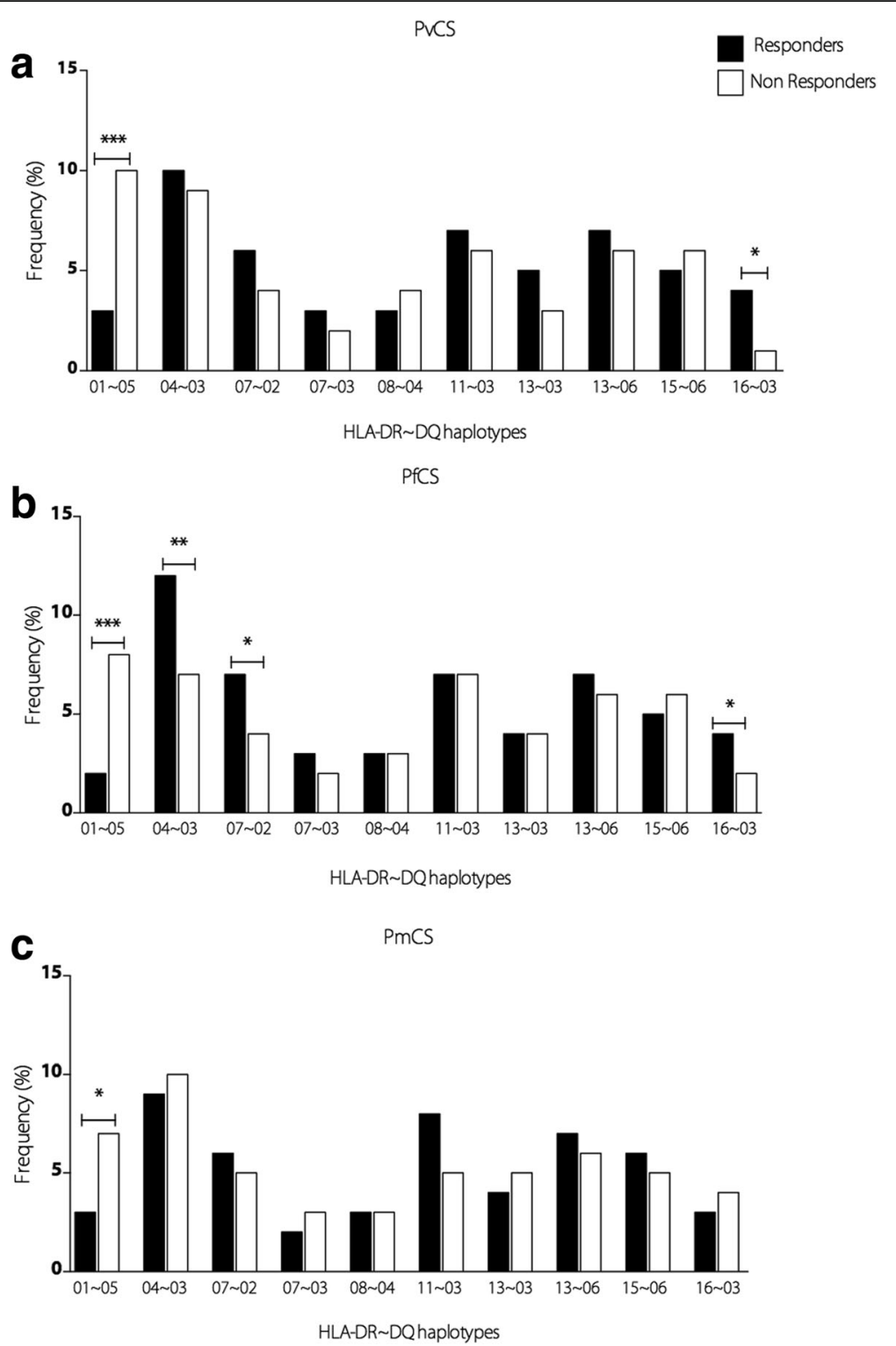

Fig. 4 Association of HLA DRB1*DQB1 haplotypes and CS antibody response. Frequency (\%) of HLA DRB1*DQB1 haplotypes in lgG responders and non-responders to the CS peptides of a PvCS, $\mathbf{b}$ PfCS, and $\mathbf{c}$ PmCS. HLA DRB1*DQB1 haplotypes with less than eight occurrences among subjects were not included in the analysis. These haplotypes were considered more frequent

density, asymptomatic infection, and there is a risk of recurrence decades after initial exposure, even when the infected individuals have left the endemic region [40-42]. Even though P. malariae reported cases in Rondonia have been rare, the high prevalence of antibodies to the CSP repeats of $P$. malariae in the absence of detected cases during the surveys could be due to the presence of antibodies cross-reacting with $P$. vivax, $P$. falciparum or with the simian P. brasilianum antigens [43]. Indeed, P. malariae and P. brasilianum share identical CS repeat sequences and do not segregate into distinct types in all genomic markers used so far [44].
Overall, production of anti-CSP antibodies was independent of a blood-stage infection. Current or recent blood infections appear to have little impact on seropositivity and do not seem to boost the anti-CSP response, as prevalence and levels of anti-CSP antibodies were not significantly different between parasite-positive and parasite-negative individuals. This is consistent with other studies that showed the absence of anti-CS antibody responses in individuals with confirmed exposure to $P$. falciparum, $P$. vivax and $P$. malariae [24, 45-48]. However, it also important to note that in the case of $P$. vivax, the presence of blood stage infection does not necessarily indicate 
a new infection as it may be due to a relapse. It is not known if the presence of hypnozoites/relapses could influence serological profile, especially those against sporozoites.

Numerous studies that evaluated anti-CSP antibody responses in endemic populations showed that an antisporozoite humoral immune response was independent of a blood stage infection, but correlates with exposure and increases with age [49-51]. We were unable to establish a correlation between specific anti-CSP responses and age, time of residence in endemic area, number of previous malaria infections, and time elapsed since the last malaria infection, corroborating with previous observations that anti-CSP antibodies do not necessarily correlate with recent exposure [17, 46, 47]. However, seroprevalence indicates ongoing exposure to all three Plasmodium species in the area.

As infection and exposure refer to infective bites by a mosquito regardless whether it results in a blood-stage infection, it is difficult to obtain data that reflect individual natural exposure to infective bites. On the other hand, several studies showed that anti-CSP antibody responses change with seasonal or geographical transmission intensity. In low transmission settings, anti-CSP antibodies formed at a slower rate and decayed at a faster rate compared to anti-blood-stage antibodies [21]. In contrast, half-life of antibodies against CSP has been reported as long as several years in a malaria outbreak in Brazil and in Africa, and as short as 27 days in Thailand $[25,52,53]$.

Although we were unable to establish a correlation between time since the last malaria infection and specific anti-CSP responses, the prevalence of antibodies to PvCS and PfCS was higher in individuals for whom the time elapsed since the last malaria infection was more than one year ago.

One major limitation of our study is that serological measures were performed from a single cross-sectional survey at the malaria transmission season and changes in prevalences and the persistence of antibodies to the CSP after the end of the transmission was not evaluated. Interestingly, in our study, a proportion of naturally exposed individuals remained unresponsive to all CSP repeats $(28 \%)$, even after a history of repeated malaria exposures and current $P$. falciparum and $P$. vivax infection. Non-responsiveness to CSP in a proportion of individuals living in endemic areas is a well-documented finding and several studies have reported an association between specific HLA alleles and immune response to malaria antigen $[28,54]$. In the current study, HLADRB1*01 and HLA-DQB1*05 allelic groups were associated with a lack of antibodies to PvCS and PfCS, and the haplotype DRB1*01 DQB1*05 was also associated with non-responders, including non-responders to $P$. malariae.
Interestingly, according to the Brazilian National Registry of Bone Marrow Voluntary Donors, the allelic frequency of DRB1*01 in the Amazon region is 19.73\% and in Rondônia, it is $19.79 \%$ [55]. In addition, in $99.3 \%$ and 98 . 9\% of times (Amazon region and Rondônia, respectively) that both DRB1 and DQB1 were typed, the presence of DRB1*01 was associated with DQB1*05 allele, indicating a total linkage disequilibrium. Considering that the DRB1*01 allele is frequent in the studied population and in the Amazon region, although the ethnicity of the population may impact the frequency of HLA, a great number of individuals could be non-responders. HLA-DRB1*01 was also negatively associated with repetitive regions of $P$. vivax MSP-9 antigen in the Brazilian Amazon, and HLA-DQB1*05 along with HLA-DRB1*13 has been associated with a reduced susceptibility to severe malaria in Gambian children [28, 51, 54].

In contrast, we observed a higher frequency of DRB1*07 DQB1*02 and DRB1*04 DQB1*03 haplotypes in responders to PfCS, and DRB1*16 DQB1*03 haplotype for both PfCS and PvCS. These antibody responses did not seem to be related to exposure as no difference was observed between time of residence, number of previous malaria infection, and time since the last malaria infection, and the presence of these alleles.

\section{Conclusions}

Our results show that in a low transmission setting, naturally acquired antibody responses against the CSP repeats of $P$. vivax, $P$. falciparum, and $P$. vivax measured in a single cross-sectional study may not represent a valuable marker for monitoring recent malaria exposure, especially in an area with a high prevalence of $P$. vivax. Furthermore, HLA class II alleles (HLA-DRB1*01, HLA-DQB1*02, and HLA-DQB1*05) were associated with antibody response for CSP of $P$. vivax and P. falciparum, and it will be of interest to consider a HLA analysis when using serosurveillance among genetically diverse populations. In the context of malaria elimination, factors affecting the acquisition and maintenance of antimalarial antibodies are important for the development of serosurveillance tools.

\section{Additional file}

Additional file 1: Multilingual abstracts in the six official working languages of the United Nations. (PDF $666 \mathrm{~kb}$ )

\footnotetext{
Abbreviations

CS: Circumsporozoite; CSP: Circumsporozoite protein; EIR: Entomological inoculation rate; ELISA: Enzyme-linked immunosorbent assay; HLA: Human leukocyte antigen; IgG: Immunoglobulin G; OD: Optical density; PCR: Polymerase chain reaction; PfCS: Circumsporozoite protein repeats of $P$. falciparum; PmCS: Circumsporozoite protein repeats of $P$. malariae; PVCS: Circumsporozoite protein repeats of $P$. vivax; RDT: Rapid diagnostic test; RI: Reactivity index; SD: Standard deviation
} 


\section{Acknowledgments}

We thank the Secretary of Health of Rondônia state and the Laboratório Central - LACEN of Rondônia for fieldwork support. We are grateful to all individuals who participated for their cooperation and generous donation of blood, which made this study possible.

\section{Funding}

This work was supported by grants from PRONEX Rede Malaria, Conselho Nacional de Pesquisa e Tecnologia (CNPq) (5555659/2009-7) and Fundação de Amparo à Pesquisa do Rio de Janeiro (E-26/170.003/2010). JO-F is a recipient of research productivity fellowships from the CNPq (307659/2016-0). VAP is the recipient of a CNPq fellowship (142104/2014-0). The funders had no role in the study design, data collection and analysis, decision to publish, or preparation of the paper.

\section{Authors' contributions}

JO-F conceived the study, participated in its design, coordinated the field survey and sample collection, and helped to draft and revise the paper. VAP participated in the study design, carried out the ELISA assays, performed the statistical analysis, and wrote the paper. JCS-A and MPAV collected the data during the field surveys and laboratory processed field-collected samples. MPAV, AT, DP-d-S, DMB, and JO-F conducted the fieldwork and sample collection. DP-d-S, DMB, and LHdC performed the parasitological diagnosis by microscopy and PCR. LCdMSP, MTQM, and ARF performed the HLA experiments. VAP, JCS-A, and LdSV performed the antibody assays. VAP analysed the data, with support from JCS-A and JO-F. All authors read and approved the final version of this paper for publication.

\section{Ethics approval and consent to participate}

The protocol of this study was reviewed and approved by the Fundação Oswaldo Cruz Ethical Committee (CEP/FIOCRUZ, 492/08). Informed consent was obtained from all participants.

\section{Competing interests}

The authors declare that they have no competing interests.

\section{Author details}

'Laboratory of Immunoparasitology, Oswaldo Cruz Institute/Fiocruz, Av. Brasil 4365, Manguinhos, Rio de Janeiro, Brazil. Institute of Infectology Emilio Ribas, São Paulo, São Paulo, Brazil. "'Laboratory of Immunodiagnosis, Departament of Biological Sciences, National School of Public Health/Fiocruz, Rio de Janeiro, Brazil. ${ }^{4}$ Laboratory of Clinical Immunology, Oswaldo Cruz Institute/Fiocruz, Rio de Janeiro, Brazil. ${ }^{5}$ Laboratory of Histocompatibility and Cryopreservation - HLA of DHE/IBRAG/UERJ, Rio de Janeiro, Brazil. ${ }^{6}$ Molecular Biology and Malaria Immunology Research Group, Centro de Pesquisas René Rachou/Fiocruz, Belo Horizonte, Minas Gerais, Brazil.

\section{Received: 11 July 2017 Accepted: 19 April 2018}

Published online: 14 May 2018

\section{References}

1. Organization WH. World malaria report 2016. Available at: http://www.who. int/malaria/publications/world-malaria-report-2016/report/en/. Accessed 24 Apr 2017.

2. Pan American Health Organization. Report on the situation of Malaria in the Americas, 2014. Washington, D.C. : PAHO; 2016. ISBN: 978-92-75-11928-0.

3. Saúde Md. Governo lança Plano de Eliminação da Malária no Brasil. Available at: http:/www.brasil.gov.br/saude/2015/11/governo-lanca-planode-eliminacao-da-malaria. Accessed 26 Apr 2017.

4. Scopel KK, Fontes CJ, Nunes AC, Horta MF, Braga EM. High prevalence of Plasmodium malariae infections in a Brazilian Amazon endemic area (Apiacas-Mato Grosso state) as detected by polymerase chain reaction. Acta Trop. 2004;90:61-4.

5. Cavasini MT, Ribeiro WL, Kawamoto F, Ferreira MU. How prevalent is Plasmodium malariae in Rondonia, western Brazilian Amazon? Rev Soc Bras Med Trop. 2000;33:489-92.

6. Siqueira AM, Mesones-Lapouble $\mathrm{O}$, Marchesini $\mathrm{P}$, et al. Plasmodium vivax landscape in Brazil: scenario and challenges. Am J Trop Med Hyg. 2016;95:87-96.

7. Ferreira MU, Castro MC. Challenges for malaria elimination in Brazil. Malar J. 2016;15:284
8. Mueller I, Galinski MR, Baird JK, et al. Key gaps in the knowledge of Plasmodium vivax, a neglected human malaria parasite. Lancet Infect Dis. 2009;9:555-66.

9. Jimenez A, Rees-Channer RR, Perera R, et al. Analytical sensitivity of current best-in-class malaria rapid diagnostic tests. Malar J. 2017;16:128.

10. Drakeley C, Schellenberg D, Kihonda J, et al. An estimation of the entomological inoculation rate for Ifakara: a semi-urban area in a region of intense malaria transmission in Tanzania. Tropical Med Int Health. 2003;8:767-74.

11. Oesterholt MJ, Bousema JT, Mwerinde OK, et al. Spatial and temporal variation in malaria transmission in a low endemicity area in northern Tanzania. Malar J. 2006;5:98.

12. Hanscheid T, Grobusch MP. How useful is PCR in the diagnosis of malaria? Trends Parasitol. 2002;18:395-8.

13. Kagan IG, Mathews H, Sulzer AJ. The serology of malaria: recent applications. Bull N Y Acad Med. 1969;45:1027-42.

14. Bruce-Chwatt L. Seroepidemiology of malaria. J Parasitol. 1970;56:552-5.

15. Bruce-Chwatt L, Draper CC, Konfortion P. Seroepidemiological evidence of eradication of malaria from Mauritius. Lancet. 1973;2:547-51.

16. Cunha MG, Silva ES, Sepulveda N, et al. Serologically defined variations in malaria endemicity in Para state. Brazil PLoS One. 2014;9:e113357.

17. Drakeley $\mathrm{CJ}$, Corran PH, Coleman PG, et al. Estimating medium- and longterm trends in malaria transmission by using serological markers of malaria exposure. Proc Natl Acad Sci U S A. 2005;102:5108-13.

18. Dewasurendra RL, Dias JN, Sepulveda N, et al. Effectiveness of a serological tool to predict malaria transmission intensity in an elimination setting. BMC Infect Dis. 2017;17:49.

19. Bousema T, Drakeley C, Gesase S, et al. Identification of hot spots of malaria transmission for targeted malaria control. J Infect Dis. 2010;201:1764-74.

20. Bwire R, Slootman EJ, Verhave JP, Bruins J, Docters van Leeuwen WM. Malaria anticircumsporozoite antibodies in Dutch soldiers returning from sub-Saharan Africa. Tropical Med Int Health. 1998;3:66-9.

21. Kusi KA, Bosomprah S, Dodoo D, et al. Anti-sporozoite antibodies as alternative markers for malaria transmission intensity estimation. Malar J. 2014;13:103.

22. Kusi KA, Bosomprah S, Kyei-Baafour E, et al. Seroprevalence of antibodies against Plasmodium falciparum Sporozoite antigens as predictive disease transmission markers in an area of Ghana with seasonal malaria transmission. PLoS One. 2016;11:e167175.

23. Nothdurft HD, Jelinek T, Bluml A, von Sonnenburg F, Loscher T. Seroconversion to circumsporozoite antigen of Plasmodium falciparum demonstrates a high risk of malaria transmission in travelers to East Africa. Clin Infect Dis. 1999;28:641-2.

24. Webster HK, Gingrich JB, Wongsrichanalai C, et al. Circumsporozoite antibody as a serologic marker of Plasmodium falciparum transmission. Am J Trop Med Hyg. 1992;47:489-97.

25. Braga EM, Fontes CJ, Krettli AU. Persistence of humoral response against sporozoite and blood-stage malaria antigens 7 years after a brief exposure to Plasmodium vivax. J Infect Dis. 1998;177:1132-5.

26. Longley RJ, Reyes-Sandoval A, Montoya-Diaz E, et al. Acquisition and longevity of antibodies to Plasmodium vivax Preerythrocytic antigens in western Thailand. Clin Vaccine Immunol. 2015;23:117-24.

27. Johnson AH, Leke RG, Mendell NR, et al. Human leukocyte antigen class II alleles influence levels of antibodies to the Plasmodium falciparum asexualstage apical membrane antigen 1 but not to merozoite surface antigen 2 and merozoite surface protein 1. Infect Immun. 2004;72:2762-71.

28. Lima-Junior JC, Rodrigues-da-Silva RN, Banic DM, et al. Influence of HLADRB1 and HLA-DQB1 alleles on IgG antibody response to the P. vivax MSP1, MSP-3alpha and MSP-9 in individuals from Brazilian endemic area. PLoS One. 2012;7:e36419.

29. Storti-Melo LM, da Costa DR, Souza-Neiras WC, et al. Influence of HLA-DRB-1 alleles on the production of antibody against CSP, MSP-1, AMA-1, and DBP in Brazilian individuals naturally infected with Plasmodium vivax. Acta Trop. 2012;121:152-5.

30. Oliveira-Ferreira J, Pratt-Riccio LR, Arruda M, et al. HLA class II and antibody responses to circumsporozoite protein repeats of $P$. vivax (VK210, VK247 and P. Vivax-like) in individuals naturally exposed to malaria. Acta Trop. 2004;92:63-9.

31. Nardin EH, Oliveira GA, Calvo-Calle JM, et al. Synthetic malaria peptide vaccine elicits high levels of antibodies in vaccinees of defined HLA genotypes. J Infect Dis. 2000;182:1486-96.

32. Arruda ME, Zimmerman RH, Souza RM, Oliveira-Ferreira J. Prevalence and level of antibodies to the circumsporozoite protein of human 
malaria parasites in five states of the Amazon region of Brazil. Mem Inst Oswaldo Cruz. 2007:102:367-71.

33. Lourenço-de-Oliveira RGA, Arlé M, da Silva TF, Castro MG, Motta MA, Deane LM. Anopheline species, some of their habits and relation to malaria in endemic areas of Rondônia state, Amazon region of Brazil. Mem Inst Oswaldo Cruz. 1989:84:501-14.

34. Deane LM, Daniel Ribeiro C, Lourenco de Oliveira R, Oliveira-Ferreira J, Guimaraes AE. Study on the natural history of malaria in areas of the Rondonia state-Brazil and problems related to its control. Rev Inst Med Trop Sao Paulo. 1988;30:153-6.

35. Tadei WP, Dutary TB. Malaria vectors in the Brazilian amazon: Anopheles of the subgenus Nyssorhynchus. Rev Inst Med Trop Sao Paulo. 2000;42:87-94.

36. National Programe for malaria Prevention and Control MoHoBA. Available at: http://portalms.saude.gov.br/saude-de-a-z/malaria/acoes-do-programade-malaria. Accessed 24 Apr 2017.

37. Snounou G, Viriyakosol S, Zhu XP, et al. High sensitivity of detection of human malaria parasites by the use of nested polymerase chain reaction. Mol Biochem Parasitol. 1993;61:315-20.

38. Nardin EH, Nussenzweig RS, Altszuler R, et al. Cellular and humoral immune responses to a recombinant $P$. falciparum CS protein in sporozoite-immunized rodents and human volunteers. Bull World Health Organ. 1990;68(Suppl):85-7.

39. R Core Team. R: A language and environment for statistical computing. $R$ Foundation for Statistical Computing, Vienna, Austria. 2014. http://www.Rproject.org/.

40. Chadee DD, Tilluckdharry CC, Maharaj P, Sinanan C. Reactivation of Plasmodium malariae infection in a Trinidadian man after neurosurgery. $\mathrm{N}$ Engl J Med. 2000;342:1924.

41. Chadee DD, Beier JC, Doon R. Re-emergence of Plasmodium malariae in Trinidad, West Indies. Ann Trop Med Parasitol. 1999;93:467-75.

42. Siala E, Khalfaoui M, Bouratbine A, Hamdi S, Hili K, Aoun K. Relapse of Plasmodium malariae malaria 20 years after living in an endemic area. Presse Med. 2005;34:371-2.

43. Nahrendorf W, Scholzen A, Sauerwein RW, Langhorne J. Cross-stage immunity for malaria vaccine development. Vaccine. 2015;33:7513-7.

44. Guimaraes LO, Bajay MM, Wunderlich $\mathrm{G}$, et al. The genetic diversity of Plasmodium malariae and Plasmodium brasilianum from human, simian and mosquito hosts in Brazil. Acta Trop. 2012;124:27-32.

45. Del Giudice G, Engers HD, Tougne C, et al. Antibodies to the repetitive epitope of Plasmodium falciparum circumsporozoite protein in a rural Tanzanian community: a longitudinal study of 132 children. Am J Trop Med Hyg. 1987;36:203-12

46. Burkot TR, Graves PM, Wirtz RA, et al. Differential antibody responses to Plasmodium falciparum and $P$. vivax circumsporozoite proteins in a human population. J Clin Microbiol. 1989;27:1346-51.

47. Project KM. The level of anti-sporozoite antibodies in a highly endemic malaria area and its relationship with exposure to mosquitoes. Trans R Soc Trop Med Hyg. 1992;86:499-504.

48. Campo JJ, Whitman TJ, Freilich D, Burgess TH, Martin GJ, Doolan DL. Toward a surrogate marker of malaria exposure: modeling longitudinal antibody measurements under outbreak conditions. PLoS One. 2011;6:e21826.

49. Del Giudice G, Lambert PH, Mendis K, Pessi A, Tanner M. Antibody responses to Plasmodium falciparum and $P$. vivax sporozoites in areas with stable and unstable malaria. Bull World Health Organ. 1990;68(Suppl):191-6.

50. Esposito F, Lombardi S, Modiano D, et al. Immunity to Plasmodium sporozoites: recent advances and applications to field research. Parassitologia. 1986;28:101-5.

51. Tapchaisri P, Asavanich A, Limsuwan S, Tharavanij S, Harinasuta KT. Antibodies against malaria sporozoites in patients with acute uncomplicated malaria and patients with cerebral malaria. Am J Trop Med Hyg. 1985;34:831-6.

52. Druilhe P, Pradier O, Marc JP, Miltgen F, Mazier D, Parent G. Levels of antibodies to Plasmodium falciparum sporozoite surface antigens reflect malaria transmission rates and are persistent in the absence of reinfection. Infect Immun. 1986;53:393-7.

53. Webster HK, Boudreau EF, Pang LW, Permpanich B, Sookto P, Wirtz RA Development of immunity in natural Plasmodium falciparum malaria: antibodies to the falciparum sporozoite vaccine 1 antigen (R32tet32). J Clin Microbiol. 1987;25:1002-8.

54. Hill AV, Allsopp CE, Kwiatkowski D, et al. Common west African HLA antigens are associated with protection from severe malaria. Nature. 1991;352:595-600.

55. REDOME. Available at: http://redome.inca.gov.br/o-redome/. Accessed 30 Aug 2017.

\section{Ready to submit your research? Choose BMC and benefit from:}

- fast, convenient online submission

- thorough peer review by experienced researchers in your field

- rapid publication on acceptance

- support for research data, including large and complex data types

- gold Open Access which fosters wider collaboration and increased citations

- maximum visibility for your research: over $100 \mathrm{M}$ website views per year

At BMC, research is always in progress.

Learn more biomedcentral.com/submissions 